CASE REPORT

\title{
Cystadenofibroma of Ovary: A Rare Case Report
}

\author{
Pratibha Singh ${ }^{1}$, Meenakshi Gothwal ${ }^{2}$, Garima Yadav ${ }^{3}$, Navdeep K Ghuman ${ }^{4}$
}

\begin{abstract}
Ovarian cystadenofibroma of a rare benign tumor of the ovary often mimicking as a malignant ovarian tumor. Cystadenofibroma of the ovary affects females in the fifth decade, usually a benign tumor. Their presentation often mimics a malignant tumor posing a difficult management decision for the clinicians. It has epithelial and fibrous stromal components; they represent less than $2 \%$ of benign ovarian tumors. We report here a case of a postmenopausal woman with clinical presentation of a mass per abdomen, suspicious of malignant ovarian tumor based on imaging of complex cysts with thick septa and a raised CA-125. She underwent exploratory laparotomy for the same, and histopathology revealed it to be a benign serous cystadenofibroma of the ovary. We report this case for its rarity as well as for certain features that may help a clinician to suspect this entity intraoperatively, and optimize management for this benign condition.

Keywords: Laparotomy, Ovarian cystadenofibroma, Ovarian tumor.

Journal of South Asian Federation of Menopause Societies (2019): 10.5005/jp-journals-10032-1191
\end{abstract}

\section{INTRODUCTION}

Tumors of ovary can be benign, borderline, or malignant. Their distinction preoperatively is important as it helps in optimal surgical planning, avoiding unnecessary extensive interventions, as well as not compromising the optimal surgical cure.

Benign tumors generally require observation or limited surgery, which can be achieved laparoscopically. Borderline or low malignant potential (LMP) tumors-as the name suggests-are malignant tumors that require an optimal surgical procedure but generally do not recur after optimal surgery. Malignant ovarian tumors spread and metastasize and may recur back after surgery depending on the stage of disease and require extensive dissection.

Ovarian cystadenofibroma is a rare benign tumor, occurring in females aged $15-65$ years ${ }^{1}$ and occur in $1.7 \%$ of benign ovarian tumors. ${ }^{1}$ It is a slow-growing epithelial tumor. It has cystic and solid masses within the ovary and can present as a single or multiple masses in the ovary often making the diagnosis of benign or malignant all the more difficult. This tumor mostly presents as single mass within the ovary or less commonly as multiple masses within a single ovary. Rarely it may affect bilateral ovaries. These tumors can be asymptomatic or may present with vague pain and heaviness, sometimes as a lump in the lower abdomen. Ultrasonography generally shows a multicystic appearance with solid and cystic components; MRI may be more helpful. ${ }^{2}$ Tumor markers may not be of much help in differentiating benign or malignant ovarian tumors. These patients often require surgical treatment. These tumors have a good prognosis with adequate surgical treatment.

\section{Case Description}

A 51-year-old multiparous lady came with complaints of lump in abdomen and vague lower abdominal heaviness for the last 2 months to the gynecology OPD. She was P3003 and menopausal for the last 3 years. There was no history of loss of weight or appetite and no family history of malignancy. Examination revealed a stable vitals, and an abdominal mass of 18 weeks' size, soft to firm with variegated feel, and nontender. Free fluid was present, which could be elicited by shifting dullness in the abdomen. Per speculum

\author{
${ }^{1-4}$ Department of Obstetrics and Gynecology, All India Institute of \\ Medical Sciences, Jodhpur, Rajasthan, India \\ Corresponding Author: Pratibha Singh, Department of Obstetrics and \\ Gynecology, All India Institute of Medical Sciences, Jodhpur, Rajasthan, \\ India, Phone: +91 731-2740122, e-mail: drpratibha69@hotmail.com \\ How to cite this article: Singh P, Gothwal M, Yadav G, et al. \\ Cystadenofibroma of Ovary: A Rare Case Report. J South Asian Feder \\ Menopause Soc 2019;7(2):94-96. \\ Source of support: Nil \\ Conflict of interest: None
}

examination was normal; per vaginal examination revealed a normal-size uterus pushed to the left side and a mass of size $15 \times 20$ $\mathrm{cm}$ separate from the uterus. Sonography was done, which showed a multiple cystic and solid areas in the right adnexa, with thick septa suggestive of a complex cyst and free fluid in the abdomen. CA-125 was $45 \mathrm{mIU} / \mathrm{L} ;$ CA-19.9 and CEA were within normal limits.

A possibility of malignant ovarian tumor was kept and MRI was done, which showed low signal intensity on T2-weighted images and many septations with a possibility of a malignant ovarian tumor. Exploratory laparotomy was done after routine preoperative investigations. Total abdominal hysterectomy and bilateral salpingo-oophorectomy, exploration of abdominal organs, and infracolic omentectomy were done.

The abdomen was opened by midline vertical incision and straw-colored appx $150 \mathrm{~cm}^{3}$ of free fluid was present, which was sent for cytology. The uterus, one-sided tube, and ovary were normal in appearance; a big ovarian mass of $15 \times 18 \mathrm{~cm}$ approximately with multiple cystic tumors was seen along with some normal-looking ovarian tissue on the other side (Fig. 1). These tumors were seen as projections from the surface of the ovary (Fig. 2). No peritoneal deposits were seen anywhere in the abdomen and the omentum was also free from any tumor deposits. Her postoperative period in the hospital was uneventful. She was doing well after 2 months of follow-up.

Histopathology of the mass was benign ovarian cyst adenofibroma.

(c) The Author(s). 2019 Open Access This article is distributed under the terms of the Creative Commons Attribution 4.0 International License (https://creativecommons. org/licenses/by-nc/4.0/), which permits unrestricted use, distribution, and non-commercial reproduction in any medium, provided you give appropriate credit to the original author(s) and the source, provide a link to the Creative Commons license, and indicate if changes were made. The Creative Commons Public Domain Dedication waiver (http://creativecommons.org/publicdomain/zero/1.0/) applies to the data made available in this article, unless otherwise stated. 


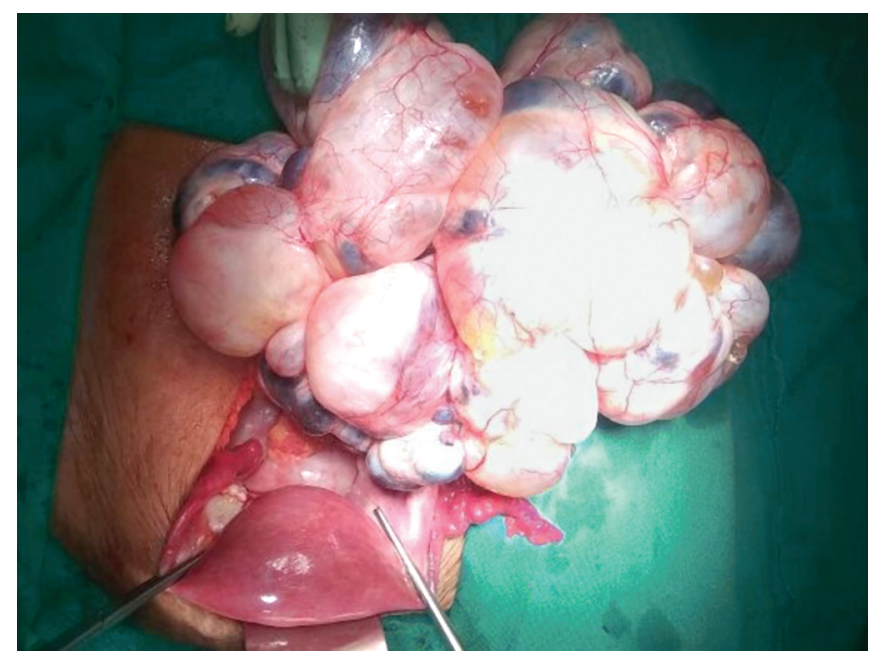

Fig. 1: Intraoperative specimen of the ovarian tumor and the uterus with normal opposite-side adnexa

\section{Discussion}

Benign ovarian tumors are of wide variety; cystadenoma is common but cystadenofibroma is rare. The epithelial and the stromal components are the major constituents, and their relative proportions determine the solid, semisolid, or the fluid state of the tumor. These tumors are classified according to the epithelial cell type present as serous, mucinous, endometroid, clear cell, and mixed categories. Serous cystadenofibroma are the commonest of the adenofibromas; other variety being endometroid, mucinous, and clear cell type. ${ }^{3}$ The degree of epithelial proliferation and its relation to the stromal component of the tumor are the criteria used for the classification as benign, borderline, or malignant, although most of the reported ovarian cystadenofibromas were benign. ${ }^{1,4}$

The clinical presentation of these tumors is generally vague, and usually occurs in fourth and fifth decade of life; they present with pain abdomen, distention of abdomen, lump in abdomen, or urinary and/or rectal symptoms may also occur. Earlier it was reported commonly in women who had DES exposure in utero. ${ }^{5}$ Rarely these tumor may present with vaginal bleeding and feminization if the tumor also had hormonal (estrogen) secretion, which was proposed to be coming from excessive stromal activity by few researchers. ${ }^{1,6}$ However, their effect on endometrium was not proved by some others. ${ }^{6-8}$

These tumors generally have favorable outcome even after conservative surgery documenting a benign nature of these tumors. They have extremely rare malignant potential.

Preoperative diagnosis is difficult as these tumors mimic malignancy due to their multicystic nature, thick septa, and solid component; even tumor markers may not be of much help. Rarely these tumors grow beyond $20 \mathrm{~cm}$; they are multiloculated with papillary projections from the ovary. ${ }^{6}$ Imaging and gross appearance of these tumors are generally suggestive of malignancy or tumors of low malignant potential. MRI can be helpful as the fibrous component frequently gives a characteristic appearance of low-signal intensity on T2W images that may help differentiating it from malignant ovarian tumors. 5,9

The frozen section may be of some help intraoperatively, though definitive diagnosis of benign may not be always be possible. ${ }^{10}$

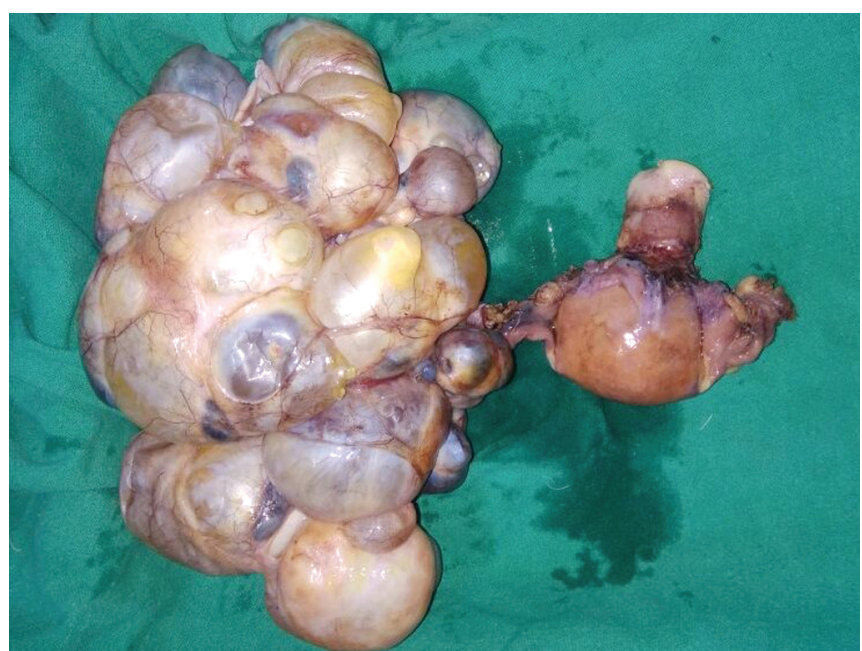

Fig. 2: Postoperative specimen of the ovarian tumor-ovarian cystadenofibroma [histopathological examination (HPE) diagnosis]

Laparoscopy has been used for removal of benign ovarian cysts, but their role in these tumors is not well documented. Even diagnostic laparoscopy may not be of much help as grossly also these tumors mimic malignancy. Few case repots are there where these tumors have been removed laparoscopically by decompression and without spill. ${ }^{7}$ However, if the solid component is more it may not be possible to remove it intact or without spill in the peritoneal cavity.

In the absence of definitive diagnostic clue, it becomes important to have a detailed dialogue with the patient and her relative preoperatively for the nature of mass and the various treatment options available to her.

In our case, a detailed discussion with the patient regarding the nature of mass, imaging findings, and our limited ability to conclusively reach to a diagnosis was helpful in accepting the diagnosis by the patient and her relatives as well as in further treatment planning. A preoperative imaging (MRI), normal tumor markers, gross appearance of the tumor, and normal intra-abdominal appearance should raise suspicion in a keen clinician; if facility for the frozen section is also used, it may help in intraoperative diagnosis avoiding extensive surgery.

\section{Conclusion}

Cystadenofibroma are benign tumors with a very good prognosis after complete removal from ovaries. Our inability to diagnose it preoperatively is due to lack of characteristic features on imaging. Diagnostic laparoscopy might may not be of much help as grossly these tumor appear malignant. Being benign, these tumors have a very low recurrence risk on compete removal through surgery.

\section{References}

1. Czernobilsky B, Borenstein R, Lancet M. Cystadenofibroma of the ovary. A clinicopathologic study of 34 cases and comparison with serous cystadenoma. Cancer 1974;34(6):1971-1981. DOI: 10.1002/1097-0142(197412)34:63.0.co;2-n.

2. Jung DC, Kim SH, Kim SH. MR imaging findings of ovarian cystadenofibroma and cystadenocarcinofibroma: clues for differential diagnosis. Korean J Radiol 2006;7(3):199-204. DOI: 10.3348/kjr.2006.7.3.199. 
3. Wolfe SA, Seckinger DL. Various anatomical types of ovarian adenofibroma. Am J Obstet Gynecol 1967;99(1):121-125. DOI: 10.1016/ s0002-9378(16)34500-8.

4. Compton HL, Finck FM. Serous adenofibroma and cystadenofibroma of the ovary. Obstet Gynecol 1970;36(4):636-645.

5. Schmidt G, Fowler WC. Ovarian cystadenofibroma in three women with antenatal exposure to diethylstilbestrol. Gynecol Oncol 1982;14(2):175-184. DOI: 10.1016/0090-8258(82)90087-7.

6. Mc Nulty JR. The ovarian serous cystadenofibroma - A report of 25 cases. Am J Obstet Gynecol 1999;77:133847.

7. Sills ES, Kaplan CR, Perloe M, et al. Laparoscopic approach to an uncommon adnexal neoplasm associated with infertility: serous cystadenofibroma of the fallopian tube. J Am Assoc Gynecol Laparosc 2003;10(4):545-547. DOI: 10.1016/s1074-3804(05) 60165-x.

8. Roth LM, Langley FA, Fox H, et al. Ovarian clear cell adenofibromatous tumours. Cancer 1984;53(5):1156-1163. DOI: 10.1002/10970142(19840301)53:53.0.co;2-2.

9. ChoSM, Byun JY, RhaSE, etal.CT and MRI findings of cystadenofibromas of the ovary. Eur Radiol 2004;14(5):798-804. DOI: 10.1007/s00330-0032060-z.

10. Lee $\mathrm{DH}$. A case of mucinous cystadenofibroma of the ovary. Case Rep Obstet Gynecol 2014;2014:130530. DOI: 10.1155/2014/ 130530. 